•综述・

\title{
物种共存理论研究进展
}

\author{
储诚进 $1^{*}$ 王酉石 ${ }^{1}$ 刘 宇 ${ }^{1}$ 蒋 林 $^{2}$ 何芳良 1,3 \\ 1 (中山大学-阿尔伯塔大学生物多样性保护联合实验室, 中山大学有害生物控制与资源利用国家重点实验室, \\ 中山大学生命科学学院, 广州 510275) \\ 2 (School of Biology, Georgia Institute of Technology, Atlanta, GA, USA 30332) \\ 3 (Department of Renewable Resources, University of Alberta, Edmonton, Canada T6G 2H1)
}

\begin{abstract}
摘要: 群落内的多物种如何共存是群落生态学的核心研究内容之一。经典的物种共存理论强调物种之间的生态位 分化, 注重具体共存机制的研究。这种以具体共存机制为研究对象的方法一定程度上促进了当代物种共存理论框 架的形成。在当代物种共存理论框架下, 物种间的差异被划分为两类综合性的抽象差异一一生态位差异和平均适 合度差异, 前者促进物种共存, 对应稳定化机制; 后者导致竞争排除, 对应均等化机制。本文在简要回顾经典物种 共存理论的基础上, 介绍了当代物种共存理论的框架(包括理论的形成和定义)、基于该理论的部分实验验证工作 及其在一些重要生态学问题中的应用。当代物种共存理论不仅揭示了群落内物种是如何共存的这一基本理论问题, 更重要的是在全球变化的背景下该理论对生物多样性的保护和管理具有重要的应用价值。期望本文的介绍有助于 国内生态学和生物多样性工作者了解当代物种共存理论, 并将其应用于群落构建和生物多样性维持机制等方面的 研究。
\end{abstract}

关键词：生态位；稳定化机制；均等化机制；生态位差异；平均适合度差异

\section{Advances in species coexistence theory}

\author{
Chengjin $\mathrm{Chu}^{1 *}$, Youshi Wang ${ }^{1}$, Yu Liu ${ }^{1}$, Lin Jiang ${ }^{2}$, Fangliang $\mathrm{He}^{1,3}$ \\ 1 SYSU-Alberta Joint Laboratory for Biodiversity Conservation, State Key Laboratory of Biocontrol, School of Life Sci- \\ ences, Sun Yat-sen University, Guangzhou 510275, China \\ 2 School of Biology, Georgia Institute of Technology, Atlanta, GA, USA 30332 \\ 3 Department of Renewable Resources, University of Alberta, Edmonton, Canada T6G $2 \mathrm{HI}$
}

\begin{abstract}
How species coexist locally is a fundamental question in community ecology. Classical coexistence theory underscores the importance of niche differentiation between species and focuses on specific coexistence mechanisms. Studies on these specific coexistence mechanisms have profoundly contributed to understanding species coexistence at the local scale and inspired ecologists to create a more general contemporary coexistence theory. Under the contemporary coexistence theory, species differences are categorized into two groups: niche differences and average fitness differences. Niche differences serve as stabilizing mechanisms that promote species coexistence, whereas average fitness differences are related to equalizing mechanisms that drive competitive exclusion. In this paper we provide a detailed review of contemporary coexistence theory, including its definition and theoretical models, empirical tests of these models and their applications to biodiversity studies. Coexistence theory has applications in a number of other areas including biodiversity conservation and management in a changing world beyond the basic concept of how communities are structured. We show how contemporary coexistence theory has advanced the niche-based classic coexistence theory, helping us to better understand the underlying mechanisms of community assembly and biodiversity maintenance.
\end{abstract}

Key words: niche; stabilizing mechanisms; equalizing mechanisms; niche differences; average fitness differences

收稿日期: 2017-02-12; 接受日期: 2017-04-01

基金项目: 国家自然科学基金(31622014,31570426, 31670531)

* 通讯作者 Author for correspondence. E-mail: chuchjin@mail.sysu.edu.cn 
解释多物种如何在现实群落中的共存是群落 生态学和生物多样性研究的核心内容。自2006年以 来, 国内一些有关群落构建和生物多样性维持机制 的综述对此有所介绍(周淑荣和张大勇, 2006; 牛克 昌等, 2009; 牛红玉等, 2011; 朱璧如和张大勇, 2011; 陈否等, 2014)。周淑荣和张大勇(2006)介绍了 群落中性理论的前提假设、预测能力和物种形成等 方面的研究进展, 分析了扩散限制条件下群落中性 构建中物种共存的可能机制; 牛克昌等(2009)在回 顾生态位理论和群落中性理论发展的基础上, 分析 了二者之间的主要分歧和互补性, 梳理了二者整合 的可能途径, 提出群落的多物种共存可能是随机过 程和确定性作用的共同结果, 而针对具体群落需要 深入研究二者的相对贡献; 牛红玉等(2011)综述了 群落谱系生态学的相关理论和研究进展, 讨论了如 何理解长期进化过程对群落中多物种共存的影响; 朱璧如和张大勇(2011)对Vellend (2010)提出的基于 过程的群落构建概念框架进行了介绍, 为国内工作 者理解格局与过程之间的关系提供了新视角; 陈磊 等(2014)介绍了生态位分化在物种多样性维持过程 中的作用, 提出在将来的研究中需要考虑个体分化 对局域群落构建的影响。这些综述性论文为国内从 事群落生态学和生物多样性研究的工作者理解群 落构建和生物多样性的维持机制起到了积极的推 动作用。然而, 以上综述均没有专门介绍当代物种 共存理论这一近十年来群落生态学发展最为活跃 的主题。

局域尺度上的物种共存问题是群落构建和生 物多样性维持机制研究的重要组成部分。人们常常 将物种共存、群落构建和生物多样性维持相提并 论、混淆使用, 但实际上物种共存问题与后两者的 研究存在着研究尺度上的差异。物种共存通常以较 小尺度上的群落为研究对象, 根据Vellend (2010)的 群落综合概念框架, 其侧重于非生物环境和生物选 择下群落内相互作用的组分种之间的关系, 而较少 考虑局域生态漂变的影响、物种扩散的作用以及物 种形成的贡献。简而言之, 物种共存理论主要研究 生物和非生物选择下群落组分种的生态位分化和 适合度差异。

本文首先简要回顾了经典的物种共存理论及 其局限性; 在此基础上, 介绍了Chesson (2000)提出 的当代物种共存理论, 包括理论框架的定义和部分
实验验证工作; 进而探讨当代物种共存理论在群落 生态学和全球变化生态学中的应用, 包括如何更好 地从格局来推断内在的群落构建过程和生物多样 性维持机制, 以及如何应用当代物种共存理论来预 测气候变化的影响等; 最后通过介绍当代物种共存 理论与当代生态位理论、群落构建和生物多样性维 持机制理论之间的联系，试图阐明以物种共存为核 心的群落生态学未来可能的研究方向。

\section{1 经典的物种共存理论}

经典物种共存理论强调具体的物种共存机制, 比如植物物种对土壤资源的分化利用、物种的时间 生态位分化和空间生态位分化等, 其历史可追溯到 生态位概念的提出(Grinnell, 1917)。Grinnell的生态 位概念侧重物种对外界环境条件的“需求” (需求生 态位, requirement niche), 而Elton (1927)的生态位概 念则强调物种在群落中的作用和对环境的“影响” (影响生态位, impact niche)。尽管人们对生态位概念 本身的认识存在诸多分歧(Grinnell, 1917; Elton, 1927; Gause, 1934; Hutchinson, 1957; Leibold, 1995; Chase \& Leibold, 2003; Soberón, 2007), 但是生态位 分化对于物种共存的重要性是普遍认可的。LotkaVolterra竞争模型在理论上表明了种内竞争与种间 竞争的相对强度决定了物种是稳定共存还是发生 竞争排除(Lotka, 1925; Volterra, 1926)。俄国生物学家 Gause (1934)通过草履虫实验验证了Lotka-Volterra 竞争模型的预测结果, 这意味着物种对资源的分化 利用是维持物种共存的必要条件, 即“竞争排除法 则” (Hardin, 1960)。

在 Grinnell (1917)生态位概念的影响下, Hutchinson (1957)提出了超体积生态位的概念, 即物种 的适合度是由多个因素所共同决定的，相对较为定 量地描述了物种对外界环境的需求。在这个框架下, Hutchinson试图寻找影响共存物种间相似性的因素, 其中具有重要影响的工作是关于动物体型大小的 Hutchinson比例: 当两个相似的物种共存时, 较大 的物种体型是较小物种的 1.3 倍: 竞争使得物种个体 大小发生了变化。MacArthur等在Hutchinson生态位概 念的基础上开展了一系列时至今日仍对生态学有 重要影响的研究工作(MacArthur \& Levins，1967; MacArthur, 1969, 1972; May \& MacArthur, 1972), 也促使当时的生态学家开始测量和计算物种的生 
态位宽度、生态位重叠程度等。极限相似性假说即 为一例, 其假定能够稳定共存的两个物种之间的相 似性程度存在一个上限, 超过此上限, 物种间即发 生竞争排除从而不能稳定共存(MacArthur \& Levins, 1967)。换言之, 共存物种之间的生态位不能无限重 叠。从 Grinnell (1917) 到 Hutchinson (1957) 再 到 MacArthur (1969), 都侧重于物种对环境的需求, 这 是经典物种共存理论发展的其中一条主线, 其本质 主要是以Lotka-Volterra竞争模型为基础。LotkaVolterra竞争模型是现象模型, 以竞争系数这个抽 象的指数来笼统代表具体机制对物种共存的影响 (Chase \& Leibold, 2003)。

另外一条主线以机理模型为基础, 主要是 MacArthur提出的消费者-资源模型 (MacArthur, 1972)。机理模型相对现象模型的优势在于同时考虑 了物种对环境的“需求”和“影响”, 这也是生态位概 念的一次完善, 是Grinnell生态位概念和Elton生态 位概念的综合。以机理模型为基础解释物种共存的 工作始于MacArthur (1972), 而让该模型人所共知 的是 Tilman (1980, 1982), 比如其提出的 $R$ *理论和 资源比例假说便是生态学发展史上的一个重要节 点。在机理模型中, 物种能否共存取决于 3 个方面: 资源的供给率、物种对环境的需求以及物种对环境 的影响。换言之, 物种受不同资源的限制, 且该物 种消耗最多的资源恰好是限制其增长的那个资源。

理论上, MacArthur的消费者-资源模型可以解释无 数物种在群落内的共存问题(Tilman, 1982)。然而, 这种理论上的完美性伴随的是其在实际群落中较 低的可操作性, 较难进行实验验证。

无论是基于现象模型还是基于机理模型的物 种共存理论都为生态位分化和竞争在物种共存中 的作用提供了一个相对综合性的理论框架。然而, 在20世纪80-90年代, 这两类模型都遭到了强烈的 质疑(Strong et al, 1979; Simberloff \& Boecklen, 1981; Lewin, 1983)。研究发现, 很多格局, 如Hutchinson 比例, 实际上并不具有统计上的显著性, 这意味着 生态位分化或者竞争对物种共存的作用并非像之 前假定的那么重要。因此, 人们转而倾向于认为物 种共存机制依赖于具体的群落类型。回顾群落生态 学的发展, 人们的确发现不同群落类型物种共存的 具体机制是不同的。对一些群落而言, 土壤的营养 元素含量和比例很重要(Tilman, 1982), 而在另外一
些群落中物种对光照的竞争则起着主要作用(Newman, 1973)。此外, 物种的空间生态位的分离(MacArthur, 1958)和时间生态位的分化(Chesson, 1985) 是维持某些群落物种共存的关键。同时, 对于某个 具体的群落而言, 物种共存与否是多个因素共同作 用的结果，包括因素的独立影响、不同因素之间的 交互影响以及这些因素时空变异的作用。这一度让 一些生态学家陷入了绝望的境地: 关于物种共存机 制还存在普适性的理论框架吗(Lawton, 1999)?

因此, 在 20 世纪末群落生态学中是否存在普适 性的理论成为了争论的焦点。回溯这次争论, 我们 发现群落生态学迎来了新一轮的快速发展。

\section{2 当代物种共存理论}

当代物种共存理论忽略了具体的共存机制, 转 而关注影响物种共存的一般性规律。伴随着上述争 论, 关于物种共存发展出两类相对独立的理论框 架。一是Chesson提出的关于物种共存的综合性理论 框架，也就是大家现在俗称的“当代物种共存理论” (Chesson，2000，2013); 二是群落中性理论 (Bell, 2001; Hubbell, 2001), 它忽略物种间的差异或者假 定物种间差异对于物种共存没有重要影响。尽管中 性理论有很好的拟合观测数据的能力, 但是其关键 的前提假定与人们的常识相悖。因已有多篇综述性 论文对中性理论进行过详细的介绍(周淑荣和张大 勇, 2006; 牛克昌等, 2009), 所以本文不再赘述。下 面, 我们单就Chesson的当代物种共存理论作出详 尽说明。

\section{1 生态位差异和平均适合度差异}

相对于中性理论假定物种功能等同, 当代物种 共存理论将物种之间的差异分为两类: 生态位差异 (niche difference, ND) 和平均适合度差异 (average fitness difference, AFD) (Chesson, 2000; 2013) (图1)。 生态位差异和平均适合度差异是两个非常抽象的 概念, 均不涉及具体的物种差异而是对真实的物种 差异的高度概括和抽象。对于生态位差异, 我们可 以作如下理解: 深根系物种与浅根系物种通过根系 的长短实现对资源的分化利用; 对于平均适合度差 异, 类似地我们可以将其设想为物种对相同资源在 利用效率上的差异(HilleRisLambers et al, 2012)。 由此可见, 当代物种共存理论中的生态位差异包 括了经典的物种共存理论的精髓, 即生态位分化 


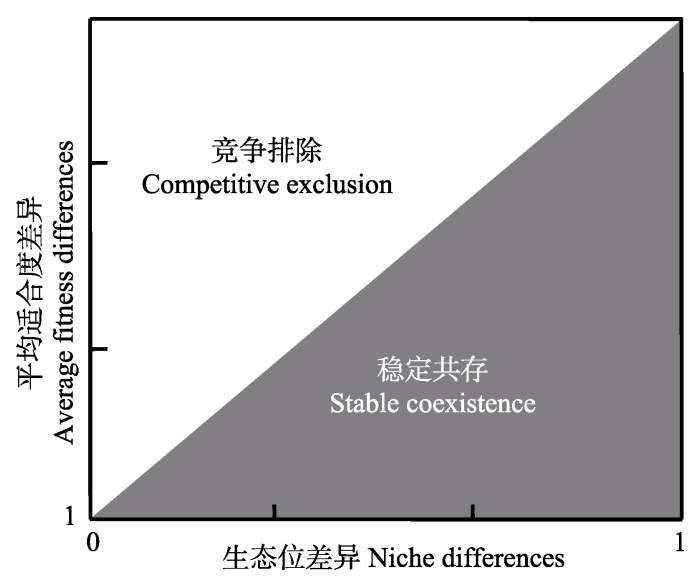

图1 当代物种共存理论框架。物种间差异分为生态位差异 和平均适合度差异, 前者促进物种共存, 后者利于竞争排 除。物种能否稳定共存取决于生态位差异和平均适合度差异 的相对大小: 灰色区域, 生态位差异大于平均适合度差 异一稳定共存; 白色区域, 平均适合度差异大于生态位差 异一竞争排除。生态位差异对应稳定化机制, 平均适合度 差异对应均等化机制。

Fig. 1 The conceptual diagram of contemporary coexistence theory. Species differences are categorized into two groups: niche differences and average fitness differences. Niche differences maintain species stable coexistence, and average fitness differences drive competitive exclusion. For a given community, the balance between niche differences and average fitness differences determines the outcome of competition. In the gray region, niche differences are larger than average fitness differences, which results in stable coexistence. In the white region, average fitness differences are stronger than niche differences, which results in competitive exclusion. Niche differences correspond to stabilizing mechanisms, and average fitness differences correspond to equalizing mechanisms.

(Letten et al, 2017)。对于稳定的物种共存而言, 生态 位差异越大越好, 而平均适合度差异则越小越好。 换言之, 生态位差异越大越有利于稳定共存, 而平 均适合度差异越大则越有利于竞争排除。对于任何 一个现实的群落, 生态位差异和平均适合度差异的 相对大小决定了物种是稳定共存还是会发生竞争 排除(Adler et al, 2007), 这是当代物种共存理论最 为核心的内容。当物种既无生态位差异也无适合度 差异的时候, 群落即为中性格局。从这个意义上来 讲, 中性理论和当代物种共存理论本质上是不冲突 的, 前者只是后者的一个特例(Adler et al, 2007)。

\section{2 理论定义}

如上所述, 生态位差异(ND)和平均适合度差异 (AFD) 是两类抽象的物种间差异, 如何量化这两类 差异是当代物种共存理论框架的关键。Chesson $(2000,2013)$ 在提出当代物种共存理论框架的同时,
给出了基于现象模型即Lotka-Volterra竞争模型的计 算公式:

$$
\begin{gathered}
\rho=1-\sqrt{\frac{\alpha_{i j} \times \alpha_{j i}}{\alpha_{j j} \times \alpha_{i i}}} \\
\frac{\kappa_{j}}{\kappa_{i}}=\sqrt{\frac{\alpha_{i i} \times \alpha_{i j}}{\alpha_{j j} \times \alpha_{j i}}}
\end{gathered}
$$

其中, $\alpha_{i i}$ 和 $\alpha_{j j}$ 为种内竞争系数, $\alpha_{i j}$ 和 $\alpha_{j i}$ 为种间竞争系 数; $\rho$ 表示生态位重叠, $1-\rho$ 即为生态位差异; $\kappa_{j} / \kappa_{i}$ 表示适合度差异 $(\kappa$ 读音为 Kappa，不同于LotkaVolterra竞争模型中的环境容纳量 $K$ )。物种稳定共存的 标准为种内竞争系数同时大于种间竞争系数: $\alpha_{i i}>\alpha_{j i}$ 并且 $\alpha_{j j}>\alpha_{i j}$ 。

Carroll等(2011)从机理模型即MacArthur的消费 者-资源模型出发, 基于种群的入侵增长率, 提出 了另外一种量化生态位差异(ND)和平均适合度差 异(AFD)的方法:

$$
\begin{aligned}
& \mathrm{ND}=1-\prod_{i=1}^{n} S_{i}^{1 / n} \\
& \mathrm{AFD}=\exp \left[\left(\overline{(\ln S)^{2}}-\overline{\ln S^{2}}\right)^{1 / 2}\right]
\end{aligned}
$$

其中, $n$ 为群落内的物种数, $S$ 表示某物种对竞争的 敏感性，通过下面的公式计算:

$$
S=1-\frac{p g r_{\text {invasion }}}{p g r_{\text {alone }}}
$$

其中, $p g r_{\text {invasion }}$ 为种群的入侵增长率, $p g r_{\text {alone }}$ 为种群 的内禀增长率。实际上, 此处的生态位差异 ND是 1 减去物种竞争敏感性 $S$ 的几何平均数, 而平均适合 度差异 $\mathrm{AFD}$ 则是物种竞争敏感性 $S$ 的几何标准差。 物种稳定共存需要满足AFD $\leq 1 /(1-\mathrm{ND})$ 。

生态位差异的取值范围为 $0-1,0$ 表示物种生态 位完全重叠, 1表示物种生态位完全分离; 平均适 合度差异的取值范围为 $\geq 1,1$ 表示物种间的适合度 相等即没有适合度的差异。因此, 当生态位差异 $\mathrm{ND}=0$ 且平均适合适度差异 $\mathrm{AFD}=1$ 的时候, 群落 即为中性。

自2000年Chesson提出当代物种共存的理论框 架之后将近 10 年左右的时间内, 该框架一直停留在 理论层面而鲜有实验验证，这可能有以下 3 个方面 的原因: 第一, 在实际工作中无论是测量竞争系数 还是种群的内禀增长率和入侵增长率都具有很大 
的挑战性，一定程度上限制了对生态位差异和平均 适合度差异的量化和理解; 第二, Hubbell于 2001年 提出的群落中性理论因其简洁性和解释力改变了 群落生态学的发展轨迹, 使得很多优秀的群落生态 学家的注意力被吸引到中性理论与生态位理论的 争论之上; 第三, Chesson于2000年发表的原始论文 相对比较晦涩, 虽然这一点并不是主要原因, 但从 整个学界的反馈来看确实也是一个不容忽视的影 响因素。

\section{3 稳定化机制和均等化机制}

物种的生态位差异导致了稳定化机制, 平均适 合度差异导致了均等化机制。如上所述, 在当代物 种共存理论当中, 稳定化机制与均等化机制共同决 定了物种能否实现稳定共存(Chesson, 2000, 2013): 稳定化机制通过物种之间的生态位分化减弱或者 消除竞争的影响, 而均等化机制通过降低物种之间 的适合度差异来促进共存。

种群增长的负密度制约或者稀有种优势是稳 定化机制的体现: 在一个稳定的群落中, 如果任何 一个物种变得特别稀少, 那么该物种的种群数量将 会表现出增加的趋势(Siepielski \& McPeek, 2010)。 在Lotka-Volterra竞争模型中, 种内竞争强于种间竞 争是生态位分化和稳定化机制的具体体现; 当且仅 当种内竞争大于种间竞争时, 物种才会实现稳定的 共存(Lotka, 1925; Volterra, 1926)。稳定化机制涵盖 了大部分经典的有关物种共存的假设和理论, 包括物 种间的时间生态位分化(Chesson, 1994)、空间生态 位分化(MacArthur, 1958)、资源分化(Tilman, 1982) 和物种特异性天敌(Janzen, 1970; Connell, 1971)等。

均等化机制是通过降低物种之间的适合度差 异或者物种在竞争能力方面的差异来促进共存的。 以对资源的竞争为例, 物种对资源的竞争能力可以 通过 $R^{*}$ 值来表示, 即某物种在群落中能够维持下去 所需的最低的资源水平(Tilman, 1982)。当多个物种 同时利用相同的限制性资源时, 具有最低 $R^{*}$ 值的物 种将竞争排除掉所有其他的物种。因此, 任何能减 少物种之间 $R^{*}$ 值差异的因子都可被归为均等化的 机制, 比如对竞争优势种的部分去除等。不同于稳 定化机制，均等化机制不会导致负密度制约。经典 物种共存理论的工作几乎都是以稳定化机制为研 究对象的, 强调物种之间的生态位分化, 却很少关 注均等化机制。实际上, 均等化机制的研究是在群
落中性理论提出后才得到了较为快速的发展的(Chave, 2004; Turnbull et al, 2008; Ostling, 2012)。

\section{4 实验验证}

对当代物种共存理论的实验验证是该理论框 架日趋成熟和为人所接受的重要一步。下面我们将 简要回顾部分验证工作，一方面阐明该领域所取得 的进展, 另一方面是希望通过研究案例的方式介绍 如何开展实验来验证当代物种共存理论。

从2.2节介绍的两类量化生态位差异和平均适 合度差异的方法可以看出, 验证的关键是获取种内 和种间竞争系数或者种群的内禀和入侵增长率。目 前针对当代物种共存理论的实验验证主要来自一 年生草地植物群落(Levine \& HilleRisLambers, 2009; Godoy et al, 2014; Godoy \& Levine, 2014; Kraft et al, 2015; Germain et al, 2016), 这主要是因为一年生植 物群落的实验周期短, 易于获取种群增长和竞争系 数的相关数据。Levine和HilleRisLambers (2009)通 过在美国加利福尼亚州的野外控制实验将理论模 型参数化, 然后在理论模型中设置种内竞争系数等 于种间竞争系数。如上所述, 稳定共存需要满足种 内竞争系数大于种间竞争系数, 所以这种处理相当 于去除了物种间生态位的差异。结果表明, 去除生 态位差异后, 能共存的物种数目明显下降, 这进一 步说明了生态位差异对于物种共存的重要性。Kraft 等(2015)利用类似的方法, 通过物种单播和两两混 种的方式获得了种内竞争系数和种间竞争系数, 进 而量化了物种间的生态位差异和平均适合度差异。 实验结果支持了当代物种共存理论的预测: 生态位 差异和平均适合度差异共同决定了物种竞争的结局。

相比于一年生草地植物群落, 当代物种共存理 论在多年生植物群落中的验证得益于长期观测数 据的积累(Adler et al, 2010; Chu et al, 2014)。Chu和 Adler (2015)收集了北美5套长期定位观测的草地野 外数据, 构建了种群统计学参数模型(包括存活率、 生长率和更新率), 然后通过积分投影模型(integral projection model)的手段计算种群入侵增长率, 进而 量化生态位差异和平均适合度差异。分析结果同样 支持了当代物种共存理论的预测, 同时还发现在这 些自然群落中物种间的生态位差异非常大而平均 适合度差异相对比较小, 这也就意味着这些多年生 草地植物群落受物种间生态位差异的强烈影响, 相 对来说非常稳定(Chu \& Adler, 2015)。 
此外, 还有研究者采用其他更易操作的实验系 统, 比如微生物系统(Zhao et al, 2016)和藻类系统 (Narwani et al, 2013)来验证当代物种共存理论。所 有这些研究都从实验的角度支持了当代物种共存 理论的预期, 也为当代物种共存理论的传播及其被 认可提供了基础。

\section{3 当代物种共存理论的应用}

当代物种共存理论在提出后先是度过了将近 10 年的沉寂期, 此后便引起了越来越多的生态学家 的兴趣和关注。这不仅仅是因为它为物种共存提供 了一个综合性、普适性的理论框架, 更为重要的是 具有非常重要的应用价值, 为解决生态学中很多关 键的科学问题都提供了新的思路和视角(Chesson, 2013)。在此, 我们列举两个方面的应用: 群落构建 机制的推断和预测气候变化的间接影响。

\section{1 基于格局推断过程}

群落生态学的一项重要任务是从群落格局推 断内在的群落构建机制。一是基于功能性状的分布 格局来探讨环境过滤和竞争在群落构建过程中的 相对作用: 如果共存物种的性状分布相对于零模型 来说表现为聚集的格局, 那么推断环境过滤是群落 构建的主要驱动力; 反之, 如果性状分布表现为发 散的格局, 那么物种之间的竞争则起着主导作用 (Ackerly \& Cornwell, 2007)。二是基于亲缘关系的谱 系群落生态学, 与基于功能性状的方法类似: 如果 生境过滤作用占主导地位, 那么相似生境将篮选出 适应能力相似、亲缘关系较近的物种, 从而表现为 谱系的聚集; 相反, 竞争排斥会使得生态位相似的 物种无法共存于同一环境, 群落内物种的亲缘关系 则较远, 表现为谱系的发散(Webb et al, 2002)。这种 从格局推断过程的方法在2002年Webb等发表其综 述性论文后被普遍采纳。同时, 在谱系群落生态学 中有一个非常常见的重要前提假定, 即亲缘关系越 近竞争越强, 亲缘关系越远竞争越弱。

然而，这种简洁的“一一对应”的关系在自然群 落中是否成立? Mayfield和Levine (2010)对此提出 了质疑: 竞争如果排除的是亲缘关系较近的物种 (如亲缘关系较近的物种具有相似的生态位需求), 那么会使群落谱系呈发散格局; 反之, 竞争若排除 的是亲缘关系较远的物种 (如亲缘关系较远的物种 在竞争能力上可能差异较大), 则会使群落谱系呈
现聚集的格局。换言之, 竞争和环境过滤都有可能 产生聚集式的格局，上述“一一对应”的关系并非是 普遍存在的(Mayfield \& Levine, 2010)。

当代物种共存理论为解决这个问题提供了最 直接和最有效的方法。在当代物种共存理论中，物 种间的差异分为生态位差异和平均适合度差异, 而 这两类差异真实地代表了物种共存的两类驱动力。 我们可以将这两类差异视为抽象的性状。如果能够 将这两类抽象性状与具体性状如根系深浅结合在 一起(谱系距离可依此类推), 找到二者之间的联系, 就能有效地揭示内在的群落构建机制(Adler et al, 2013; Kraft et al, 2015)。比如, 如果平均适合度差异 与谱系距离差异呈现负相关关系, 那么就意味着亲 缘关系越近的物种其适合度差异越大, 而平均适合 度差异越大物种间竞争越强, 越不容易稳定共存。 目前已有一些工作报道了相关的研究成果。以一年 生草本植物为研究对象, Kraft等(2015)发现在其选 择的 11 个功能性状中, 大部分功能性状与平均适合 度差异之间的相关性很强, 而与生态位差异的相关 性不明显, 这意味着这些功能性状主要体现的是物 种之间的适合度差异。以绿藻群落为研究对象, Narwani等(2013)发现物种间的谱系距离与生态位 差异和平均适合度差异均无明显相关关系, 与物种 是否共存也不相关。这表明基于谱系距离来推断竞 争的重要性并不合适, 即上述的“亲缘关系越近竞 争越强, 亲缘关系越远竞争越弱”的假定可能是不 成立的。Germain等(2016)也发现谱系距离与生态位 差异和平均适合度差异并无一一对应的关系。

\section{2 预测气候变化的间接影响}

温度和降水等环境条件既可以通过影响光合 作用和呼吸作用等对植物产生直接的影响, 也可以 通过影响生物间的相互作用如竞争产生间接的影 响(Chu et al, 2016)。这种因环境变化所导致的间接 效应已成为预测气候变化影响的重要的不确定性 来源(Gilman et al, 2010; Adler et al, 2012; Angert et al，2013)。那么如何有效地预测气候因子间接影响 的强度呢? 对该问题的回答能够为判断是否需要 考虑气候的间接影响提供依据(Adler et al, 2012; Kleinhesselink \& Adler, 2015)。由于这种间接影响是 通过种间相互作用来调节的，因此基于当代物种共 存理论, 我们可以做出如下预测: 物种间的生态位 差异越大, 物种间的竞争可能越弱, 气候的间接影 
响就越小; 而物种间的生态位差异越小, 物种间的 竞争可能越强, 则气候的间接影响越大。换言之, 可以通过物种间的生态位差异来预测气候间接影 响的强度。基于长期的野外观测数据, 结合积分投 影模型等手段, Chu等(2016)发现物种间的生态位差 异确实与气候间接影响的强度呈现一定的相关关 系: 当生态位差异很大的时候, 气候间接影响的强 度将变得很小。这也就意味着在物种间生态位差异 较大的情况下, 气候对植物群落的直接影响将强于 通过物种间相互作用所产生的间接影响, 此时就可 以忽略气候的间接影响。

\section{4 研究展望}

从前面的介绍中我们可以看到, 当代物种共存 理论为局域群落物种共存提供了一个普适性的理 论框架: 从纷繁复杂的具体的共存机制中抽象出物 种的两类差异, 生态位差异促进物种共存, 平均适 合度差异促进竞争排除。所有有关物种共存的研究 均可以放到这个框架中进行理解和解释。然而, 这 样一个“万能”的理论本身实际上也并非是“万能”的, 而是存在着一定的局限。下面我们将从 3 个方面入 手探讨如何更好地应用当代物种共存理论来解决 群落生态学家所关注的问题。

\section{1 对理论框架本身的检验}

经过近 20 年的发展, 当代物种共存理论本身已 相对成熟, 这主要体现在两个方面: 一是无论是基 于现象模型(如Lotka-Volterra模型)还是机理模型(如 消费者一资源模型), 量化物种间的生态位差异和平 均适合度差异在理论上和实践中都是可行的(Carroll et al, 2011; Chesson, 2013; Narwani et al, 2013; Godoy et al, 2014; Chu \& Adler, 2015); 二是越来越 多的群落生态学家对当代物种共存理论的接受和 认可部分源于中性理论的影响逐渐消退。然而, 正 如我们前面所提到的, 无论是测量竞争系数还是种 群入侵增长率, 在实际操作中都存在着很大的困难, 这也是为什么到目前为止针对该理论的实验验证 都主要集中在相对简单的系统, 如一年生草地植物 群落、微生物群落和藻类群落。

在当代物种共存理论中, 物种间竞争的结局有 两类：稳定共存或者竞争排除。而在自然群落中, 要观察到竞争排除或者稳定共存通常都需要相对 较长的时间, 这实际上是很难实现的。但任何一个
物种种群的增长或消亡最终都要体现在种群的统 计学参数上, 包括个体的存活率、生长率和繁殖率, 所以相对于观察稳定共存或竞争排除, 转而关注竞 争对种群统计学参数的影响是目前检验当代物种 共存理论的替代方法(Harms et al，2000; Funk \& Wolf, 2016)。这是其一。

同时, 从理论上来说, 量化生态位差异和平均 适合度差异需要测量竞争系数或者种群的入侵增 长率。而在复杂的自然系统中, 这一点同样很难实 现。为此我们同样需要寻找一个替代的方案。对于 功能性状而言, 假设有两个物种 $\mathrm{A}$ 和 $\mathrm{B}$, 其性状值分 别为 $\mathrm{T}_{\mathrm{A}}$ 和 $\mathrm{T}_{\mathrm{B}}$ 。人们提出了用物种间绝对性状距离 (性状间差异的绝对值, $\left|\mathrm{T}_{\mathrm{A}}-\mathrm{T}_{\mathrm{B}}\right|$ )来代表生态位差异, 而用物种间等级性状距离(性状间差异, $\mathrm{T}_{\mathrm{A}}-\mathrm{T}_{\mathrm{B}}$ ) 来代 表平均适合度差异。换言之, 绝对性状距离是没有 方向的, 只要存在差异就有利于物种的共存, 体现 的是物种间生态位的分化; 而等级性状距离具有方 向, 体现的是物种间适合度的差异(Kunstler et al, 2012)。这是其二。

通过上述两个方面的替代, 就可以对当代物种 共存理论开展行之有效的验证。利用法国全国林业 调查的数据, Kunstler等(2012)发现物种间的竞争强 度主要受性状等级(等级性状距离) 所驱动, 而与性 状或谱系的相似性(性状相似性即绝对性状距离)没 有明显的相关关系。Gross等(2015)在新西兰开展的 草地实验表明, 性状间的差异主要体现的是竞争的 等级, 即在该实验中所选取的性状如植株高度和特 定叶面积指数代表的主要是物种间的平均适合度 差异。

需要指出的是, 这种替代方法只是对当代物种 共存理论的一个近似验证。随着长期观测数据的积 累, 在复杂自然群落中直接检验当代物种共存理论 将会逐渐成为现实, 也为更准确和全面地揭示群落 内在的构建机制提供了可能。

\section{2 与具体物种共存机制研究的结合}

当代物种共存理论是对过去所有具体共存机 制的综合、抽象和升华, 生态位差异和平均适合度 差异是对物种功能性状的简化处理。伴随当代物种 共存理论“万能”解释能力的是对具体物种共存机制 的忽略。例如, 假定有一个自然群落, 我们知道了 其物种间生态位差异是 0.9 , 平均适合度差异是 2.6 , 那么我们能够从中得到多少有关该群落的有价值 
的信息? 很少! 再者, 这个群落主要受什么因素 的影响? 物种间的性状和谱系差异如何? 群落对 于环境条件的变化会做出什么样的响应? 都不得 而知。

将当代物种共存理论与具体共存机制相结合 是当代物种共存理论发展的必然。而具体物种共存 机制正是生态位理论的核心。生态位理论按照生态 位的定义大体上可以划分为经典生态位理论和当 代生态位理论, 这在本文的第一部分均有介绍。经 典生态位理论主要是以现象模型如Lotka-Volterra模 型为基础, 侧重物种对环境的需求。当代生态位理 论以机理模型如消费者一资源模型为基础, 既包含 了物种对环境的需求, 又包含了物种对环境的影 响。这里我们侧重当代物种共存理论与当代生态位 理论的结合, 而这两个理论正是通过生态位的概念 密切联系在一起(Kleinhesselink \& Adler, 2015; Letten et al, 2017)。在当代生态位理论中, 物种能否共 存取决于 3 个方面: 资源的供给率、物种对环境的需 求以及物种对环境的影响。那么这 3 个因素与当代 物种共存理论中的生态位差异(稳定化机制)和平均 适合度差异(均等化机制)有什么关系? Letten等 (2017)发现资源的供给率体现的是均等化的机制, 物种对环境的影响体现的是稳定化的机制, 而物种 对环境的需求可能是均等化机制也可能是稳定化 机制。这也就是说当代物种共存理论与当代生态位 理论确实是可以有机地结合在一起的; 也只有将二 者紧密结合才能最大限度地发挥二者的作用, 从而 为更全面地理解物种共存提供基础。

\section{3 与基于过程的群落构建机制研究的整合}

从格局推断过程因其不具有唯一性而显得非 常复杂和不可靠。比如前面所介绍的从功能性状或 谱系结构推断群落内在的构建机制。群落生态学在 一定程度上过于注重对格局的发现和描述, 比如物 种多度分布, 而忽视了对内在过程的深入探讨。这 解释了中性理论在其提出后的十多年里在群落生 态学的研究中占据的主导地位。如果一直按照“从 格局推断过程”这一思路发展下去, 那么群落生态 学可能就只会原地踏步。当代物种共存理论在这个 方面前进了一步, 其与功能性状和谱系关系的结合 将能更可靠地揭示出竞争的相对重要性。

Vellend (2010, 2016)提出的基于过程的群落构 建框架被认为是关于群落构建机制普适性理论的
有益尝试。朱璧如和张大勇(2011)对该理论构建框 架进行了详细的介绍。简言之, 该理论框架以过程 为出发点, 认为群落的构建由 4 个基本过程所共同 决定: 选择、漂变、扩散和成种。在不同的群落中 各个过程的相对重要性可能不同。其中, 平衡选择 (负密度制约或负频度制约)所对应的正是我们所熟 知的生态位理论和当代物种共存理论。如何将当代 物种共存理论和生态位理论与基于过程的群落构 建框架有机地结合在一起, 从而揭示群落构建和生 物多样性维持的内在机制, 是未来研究工作的一个 重要发展方向。随着当代物种共存理论和基于过程 的群落构建框架日趋成熟和完善, 群落生态学正在 迎来一个新的发展时期。

致谢：本文是在2016年“第十届海峡两岸森林动态 样区研讨会”报告的基础上整理而成，感谢中国科 学院植物研究所马克平研究员的支持和鼓励。

\section{参考文献}

Ackerly DD, Cornwell WK (2007) A trait-based approach to community assembly: partitioning of species trait values into within- and among-community components. Ecology Letters, 10, 135-145.

Adler PB, Dalgleish HJ, Ellner SP (2012) Forecasting plant community impacts of climate variability and change: when do competitive interactions matter? Journal of Ecology, 100, 478-487.

Adler PB, Ellner SP, Levine JM (2010) Coexistence of perennial plants: an embarrassment of niches. Ecology Letters, 13, 1019-1029.

Adler PB, Fajardo A, Kleinhesselink AR, Kraft NJB (2013) Trait-based tests of coexistence mechanisms. Ecology Letters, 16, 1294-1306.

Adler PB, HilleRisLambers J, Levine JM (2007) A niche for neutrality. Ecology Letters, 10, 95-104.

Angert AL, LaDeau SL, Ostfeld RS (2013) Climate change and species interactions: ways forward. Annals of the New York Academy of Sciences, 1297, 1-7.

Bell G (2001) Neutral macroecology. Science, 293, 2413-2418.

Carroll IT, Cardinale BJ, Nisbet RM (2011) Niche and fitness differences relate the maintenance of diversity to ecosystem function. Ecology, 92, 1157-1165.

Chase JM, Leibold MA (2003) Ecological Niches: Linking Classical and Contemporary Approaches. University of Chicago Press, Chicago.

Chave J (2004) Neutral theory and community ecology. Ecology Letters, 7, 241-253.

Chen L, Mi XC, Ma KP (2014) Niche differentiation and its 
consequence on biodiversity maintenance in forest communities. Chinese Bulletin of Life Sciences, 26, 112-117. (in Chinese) [陈磊, 米湘成, 马克平 (2014) 生态位分化与森 林群落物种多样性维持研究展望. 生命科学, 26, 112-117.]

Chesson P (1985) Coexistence of competitors in spatially and temporally varying environments: a look at the combined effects of different sorts of variability. Theoretical Population Biology, 28, 263-287.

Chesson P (1994) Multispecies competition in variable environments. Theoretical Population Biology, 45, 227-276.

Chesson P (2000) Mechanisms of maintenance of species diversity. Annual Review of Ecology and Systematics, 31, 343-366.

Chesson P (2013) Species competition and predation. In: Encyclopedia of Sustainability Science and Technology (ed. Meyers RA), pp. 223-256. Springer-Verlag, New York.

Chu CJ, Bartlett M, Wang YS, He FL, Weiner J, Chave J, Sack L (2016) Does climate directly influence NPP globally? Global Change Biology, 22, 12-24.

Chu CJ, Adler PB (2015) Large niche differences emerge at the recruitment stage to stabilize grassland coexistence. Ecological Monographs, 85, 373-392.

Chu CJ, Havstad KM, Kaplan N, Lauenroth WK, McClaran MP, Peters DP, Vermeire LT, Adler PB (2014) Life form influences survivorship patterns for 109 herbaceous perennials from six semi-arid ecosystems. Journal of Vegetation Science, 25, 947-954.

Connell JH (1971) On the role of natural enemies in preventing competitive exclusion in some marine animals and in rain forest trees. In: Dynamics of Populations (eds den Boer PJ, Gradwell G), pp. 298-312. Pudoc, Oosterbeek.

Elton C (1927) Animal Ecology. Sidgwick and Jackson, London.

Funk JL, Wolf AA (2016) Testing the trait-based community framework: do functional traits predict competitive outcomes? Ecology, 97, 2206-2211.

Gause GF (1934) The Struggle of Existence. Williams \& Wilkins, Baltimore.

Germain RM, Weir JT, Gilbert B (2016) Species coexistence: macroevolutionary relationships and the contingency of historical interactions. Proceedings of the Royal Society B: Biological Sciences, 283, 20160047.

Gilman SE, Urban MC, Tewksbury J, Gilchrist GW, Holt RD (2010) A framework for community interactions under climate change. Trends in Ecology \& Evolution, 25, 325-331.

Godoy O, Kraft NJB, Levine JM (2014) Phylogenetic relatedness and the determinants of competitive outcomes. Ecology Letters, 17, 836-844.

Godoy O, Levine JM (2014) Phenology effects on invasion success: insights from coupling field experiments to coexistence theory. Ecology, 95, 726-736.

Grinnell J (1917) The niche-relationships of the California thrasher. Auk, 34, 427-433.

Gross N, Liancourt P, Butters R, Duncan RP, Hulme PE (2015) Functional equivalence, competitive hierarchy and facilitation determine species coexistence in highly invaded grasslands. New Phytologist, 206, 175-186.

Hardin G (1960) The competitive exclusion principle. Science, 131, 1292-1297.

Harms K, Wright S, Calderon O, Hernandez A, Herre E (2000) Pervasive density-dependent recruitment enhances seedling diversity in a tropical forest. Nature, 404, 493-495.

HilleRisLambers J, Adler PB, Harpole WS, Levine JM, Mayfield MM (2012) Rethinking community assembly through the lens of coexistence theory. Annual Review of Ecology and Systematics, 43, 227-248.

Hubbell SP (2001) The Unified Neutral Theory of Biodiversity and Biogeography. Princeton University Press, Princeton.

Hutchinson GE (1957) Concluding remarks. Cold Spring Harbor Symposia on Quantitative Biology, 22, 415-427.

Janzen DH (1970) Herbivores and the number of tree species in tropical forests. The American Naturalist, 104, 501-528.

Kleinhesselink AR, Adler PB (2015) Indirect effects of environmental change in resource competition models. The American Naturalist, 186, 766-776.

Kraft NJB, Godoy O, Levine JM (2015) Plant functional traits and the multidimensional nature of species coexistence. Proceedings of the National Academy of Sciences, USA, 112, 797-802.

Kunstler G, Lavergne S, Courbaud B, Thuiller W, Vieilledent G, Zimmermann NE, Kattge J, Coomes DA (2012) Competitive interactions between forest trees are driven by species' trait hierarchy, not phylogenetic or functional similarity: implications for forest community assembly. Ecology Letters, 15, 831-840.

Lawton JH (1999) Are there general laws in ecology? Oikos, 84, 177-192.

Leibold MA (1995) The niche concept revisited: mechanistic models and community context. Ecology, 76, 1371-1382.

Letten AD, Ke PJ, Fukami T (2017) Linking modern coexistence theory and contemporary niche theory. Ecological Monographs, 87, 161-177.

Levine JM, HilleRisLambers J (2009) The importance of niches for the maintenance of species diversity. Nature, 461, 254-257.

Lewin R (1983) Santa Rosalia was a goat. Science, 221, 636-639.

Lotka AJ (1925) Elements of Physical Biology. Williams \& Wilkins Company, Baltimore.

MacArthur RH (1958) Population ecology of some warblers of northeastern coniferous forests. Ecology, 39, 599-619.

MacArthur RH (1969) The theory of the niche. In: Population Biology and Evolution (ed. Lewontin RC), pp. 159-176. Syracuse University Press, Syracuse.

MacArthur RH (1972) Geographical Ecology: Patterns in the 
Distribution of Species. Princeton University Press, Princeton.

MacArthur RH, Levins R (1967) The limiting similarity, convergence, and divergence of coexisting species. The American Naturalist, 101, 377-385.

May RM, MacArthur RH (1972) Niche overlap as a function of environmental variability. Proceedings of the National Academy of Science, USA, 69, 1109-1113.

Mayfield MM, Levine JM (2010) Opposing effects of competitive exclusion on the phylogenetic structure of communities. Ecology Letters, 13, 1085-1093.

Narwani A, Alexandrou MA, Oakley TH, Carroll IT, Cardinale BJ (2013) Experimental evidence that evolutionary relatedness does not affect the ecological mechanisms of coexistence in freshwater green algae. Ecology Letters, 16, 13731381.

Newman EI (1973) Competition and diversity in herbaceous vegetation. Nature, 244, 310.

Niu HY, Wang ZF, Lian JY, Ye WH, Shen H (2011) New progress in community assembly: community phylogenetic structure combining evolution and ecology. Biodiversity Science, 19, 275-283. (in Chinese with English abstract) [牛 红玉, 王峥峰, 练琚愉, 叶万辉, 沈浩 (2011) 群落构建 研究的新进展: 进化和生态相结合的群落谱系结构研究. 生物多样性, 19, 275-283.]

Niu KC, Liu YN, Shen ZH, He FL, Fang JY (2009) Community assembly: the relative importance of neutral theory and niche theory. Biodiversity Science, 17, 579-593. (in Chinese with English abstract) [牛克昌, 刘怿宁, 沈泽吴, 何芳良, 方精云 (2009) 群落构建的中性理论和生态位理论. 生 物多样性, 17, 579-593.]

Ostling A (2012) Do fitness-equalizing tradeoffs lead to neutral communities? Theoretical Ecology, 5, 181-194.

Siepielski AM, McPeek MA (2010) On the evidence for species coexistence: a critique of the coexistence program. Ecology, 91, 3153-3164.

Simberloff D, Boecklen W (1981) Santa Rosalia reconsidered: size ratios and competition. Evolution, 35, 1206-1228.

Soberón J (2007) Grinnellian and Eltonian niches and geo- graphic distributions of species. Ecology Letters, 10, 1115-1123.

Strong DR, Szyska LA, Simberloff D (1979) Tests of community-wide character displacement against null hypotheses. Evolution, 33, 897-913.

Tilman D (1980) Resource: a graphical-mechanistic approach to competition and predation. The American Naturalist, 116, 362-393.

Tilman D (1982) Resource Competition and Community Structure. Princeton University Press, Princeton.

Turnbull LA, Rees M, Purves DW (2008) Why equalising trade-offs aren't always neutral? Ecology Letters, 11, 1037-1046.

Vellend BM (2010) Conceptual synthesis in community ecology. The Quarterly Review of Biology, 85, 183-206.

Vellend M (2016) The Theory of Ecological Communities. Princeton University Press, Princeton and Oxford.

Volterra V (1926) Variations and fluctuations of the number of individuals in animal species living together. In: Animal Ecology (ed. Chapman RN) (Reprinted in 1931). McGraw Hill, New York.

Webb CO, Ackerly DD, McPeek MA, Donoghue MJ (2002) Phylogenies and community ecology. Annual Review of Ecology and Systematics, 33, 475-505.

Zhao L, Zhang QG, Zhang DY (2016) Evolution alters ecological mechanisms of coexistence in experimental microcosms. Functional Ecology, 30, 1440-1446.

Zhou SR, Zhang DY (2006) Neutral theory in community ecology. Journal of Plant Ecology (Chinese Version), 30, 868-877. (in Chinese with English abstract) [周淑荣, 张大 勇 (2006) 群落生态学的中性理论. 植物生态学报, 30, 868-877.]

Zhu BR, Zhang DY (2011) A process-based theoretical framework for community ecology. Biodiversity Science, 19, 389-399. (in Chinese with English abstract) [朱璧如, 张大勇 (2011) 基于过程的群落生态学理论框架. 生物 多样性, 19, 389-339.]

(责任编委：马克平 责任编辑：黄祥忠) 